\title{
Prediction of Earthquake Hazard by Hidden Markov Model (around Bilecik, NW Turkey)
}

Research Article

\author{
Ceren Eda Can ${ }^{1}$, Gul Ergun ${ }^{1}$, Candan Gokceoglu* \\ 1 Department of Statistics, Hacettepe University, Ankara, Turkey \\ 2 Department of Geological Engineering, Hacettepe University, Ankara, Turkey
}

Received 05 March 2014; accepted 18 April 2014

\begin{abstract}
Earthquakes are one of the most important natural hazards to be evaluated carefully in engineering projects, due to the severely damaging effects on human-life and human-made structures. The hazard of an earthquake is defined by several approaches and consequently earthquake parameters such as peak ground acceleration occurring on the focused area can be determined. In an earthquake prone area, the identification of the seismicity patterns is an important task to assess the seismic activities and evaluate the risk of damage and loss along with an earthquake occurrence. As a powerful and flexible framework to characterize the temporal seismicity changes and reveal unexpected patterns, Poisson hidden Markov model provides a better understanding of the nature of earthquakes. In this paper, Poisson hidden Markov model is used to predict the earthquake hazard in Bilecik (NW Turkey) as a result of its important geographic location. Bilecik is in close proximity to the North Anatolian Fault Zone and situated between Ankara and Istanbul, the two biggest cites of Turkey. Consequently, there are major highways, railroads and many engineering structures are being constructed in this area. The annual frequencies of earthquakes occurred within a radius of $100 \mathrm{~km}$ area centered on Bilecik, from January 1900 to December 2012, with magnitudes $(M)$ at least 4.0 are modeled by using Poisson-HMM. The hazards for the next 35 years from 2013 to 2047 around the area are obtained from the model by forecasting the annual frequencies of $M \geq 4$ earthquakes.
\end{abstract}

Keywords: Hidden Markov Model • Poisson Process • EM Algorithm • Bilecik

(c) Versita sp. z o.o.

\section{Introduction}

Earthquakes are one of the severe catastrophic natural events. They are created by the active faults, and therefore, the location of an earthquake can be estimated. However, the occurrence time of an earthquake is unpredictable and has no a known systematic pattern.

*E-mail: candan_gokceoglu@yahoo.co.uk
The magnitude and the occurrence time of an earthquake are affected by various uncontrolled natural factors. The primitive approach for the detection of temporal variation of earthquake occurrence is to count the number of earthquakes in a given period of time and then to analyze the time series by a rough calculation. Various statistical methods have been used to predict earthquake hazards for the last few decades. A traditional way for modeling the occurrences within a certain time is to define a Poisson process (PP). However, there are two major drawbacks stand out during the modeling of earthquakes by Poisson 
process. The first drawback comes from the most widely known property of Poisson distribution that is the equality of the variance and the mean of the process. In some cases, the variance can be greater than the mean and the data indicates over-dispersion. There may be some periods of time with a high rate of earthquake occurrences and some with a relatively low rate[1]. Earthquake occurrences in time display considerable over-dispersion. Therefore, Poisson process is not a suitable stochastic model for modeling the data when over-dispersion problem is observed. To overcome the problem, independent Poisson mixture model can be suggested as alternative model. The second drawback is sourced from the memorylessness property of Poisson process. The data collected from the same area in successive time intervals tends to be dependent. The dependency structure of the data violates the memorylessness property of Poisson process and therefore the model becomes insufficient for the serially dependent count data. To overcome the problem, Markov chain is widely used as an effective tool to represent the serial dependent structure in the data.

Although the deterministic functions of the Markov chains have been extensively studied on earthquake hazard analysis, hidden Markov model (HMM), which represents a probabilistic functions of finite state Markov chains, recently becomes a popular tool for the earthquake data. HMM first introduced by Baum and Petrie [2] allows for overdispersion together with the dependence between the data $[3,4]$. HMM assumes that the state of the underlying system at any time $t$ is unobservable. Therefore, the system follows a hidden process having the Markov property $[1,5,6]$. Observations are defined as the outputs of another stochastic process under the influence of hidden process. All statistical inferences are made by means of through this observable process.

As the first application of HMM for Turkish earthquake data except the presentation of Can et al. [7], the present paper aims to introduce Poisson hidden Markov model (PHMM) as a candidate model for assessing the earthquake hazard in Bilecik (NW Turkey). The model assumes that the frequencies of earthquakes occurred in specified successive time intervals are derived from the family of Poisson distribution whose rate parameter process is a homogeneous Markov Chain. Rate parameter process makes PHMM allow the studied area to have varying seismicity rate. In this paper, the observed annual frequencies of earthquakes occurred within a radius of $100 \mathrm{~km}$ area centered on Bilecik, from January 1900 to December 2012, with magnitudes $M \geq 4.0$ are used in PHMM. Then, the earthquake hazard in the area is predicted by determining the annual frequencies of earthquakes for the next 35-year period (2013-2047).
Also, the forecast results of PHMM are compared to those of homogeneous PP.

\section{Earthquake Data}

Turkey is located on one of the most active seismic regions throughout the World. In Turkey, three important earthquake sources such as the North Anatolian Fault Zone, the East Anatolian Fault Zone and the Aegean Horst-Graben System exist. For this reason, Turkey has encountered large earthquakes frequently. Due to these earthquakes, Bilecik has encountered serious damages. For example, the February 20, 1956 Inonu earthquake [8] affected a part of the study area of this study. Additionally, the August 17, 1999 Izmit earthquake caused serious damage in a large area including Bilecik and its surrounding. Bilecik is located on the between Ankara and Istanbul, two biggest cities of Turkey, and also it is close to the North Anatolian Fault Zone (Figure 1). Due to its geographic location, various transportation structures such as bridges, viaducts and tunnels have been constructed or will be constructed. For these reasons, the correct earthquake prediction for Bilecik becomes more important and hence this region is selected as the focused area.

The data source is the catalogs of Boğaziçi University, Kandilli Observatory and Earthquake Research Institute [9]. The earthquakes occurred within a radius of $100 \mathrm{~km}$ area centered on Bilecik are used, because in the majority of the strong ground motion relations suggested for tectonically active regions (e.g. [10-12], the upper bound for site- source distance is taken $100 \mathrm{~km}$, which is the range where ground motions have engineering significance[13]. Observed annual frequencies of $M \geq 4.0$ earthquakes occurred in the area from 1900 to 2012 are shown in Figure 2. Generally, few earthquakes occurred per each year except for the years 1967, 1970 and 1999. It is shown that the sample variance, $\left(S^{2}=71.9823\right)$ is larger than the sample mean, $(\bar{X}=2.2301)$. It indicates a strong over-dispersion relative to Poisson distribution. It is also seen in Figure 2 that the minimum frequency is 0 and the maximum is 71 . The median of the data is 1 and the mode is 0 . As a result of the skewness coefficient, $\left(\gamma_{1}=6.5113\right)$, the distribution of the observed data is skewed to the right. The over-dispersion and skewness of the data show that HMM is a suitable tool for the earthquake data.

The data is checked whether it follows a specific Poisson distribution, a goodness of fit test is applied. It is seen that there is insufficient evidence to conclude that the data fits a single Poisson distribution. It is generated from several 

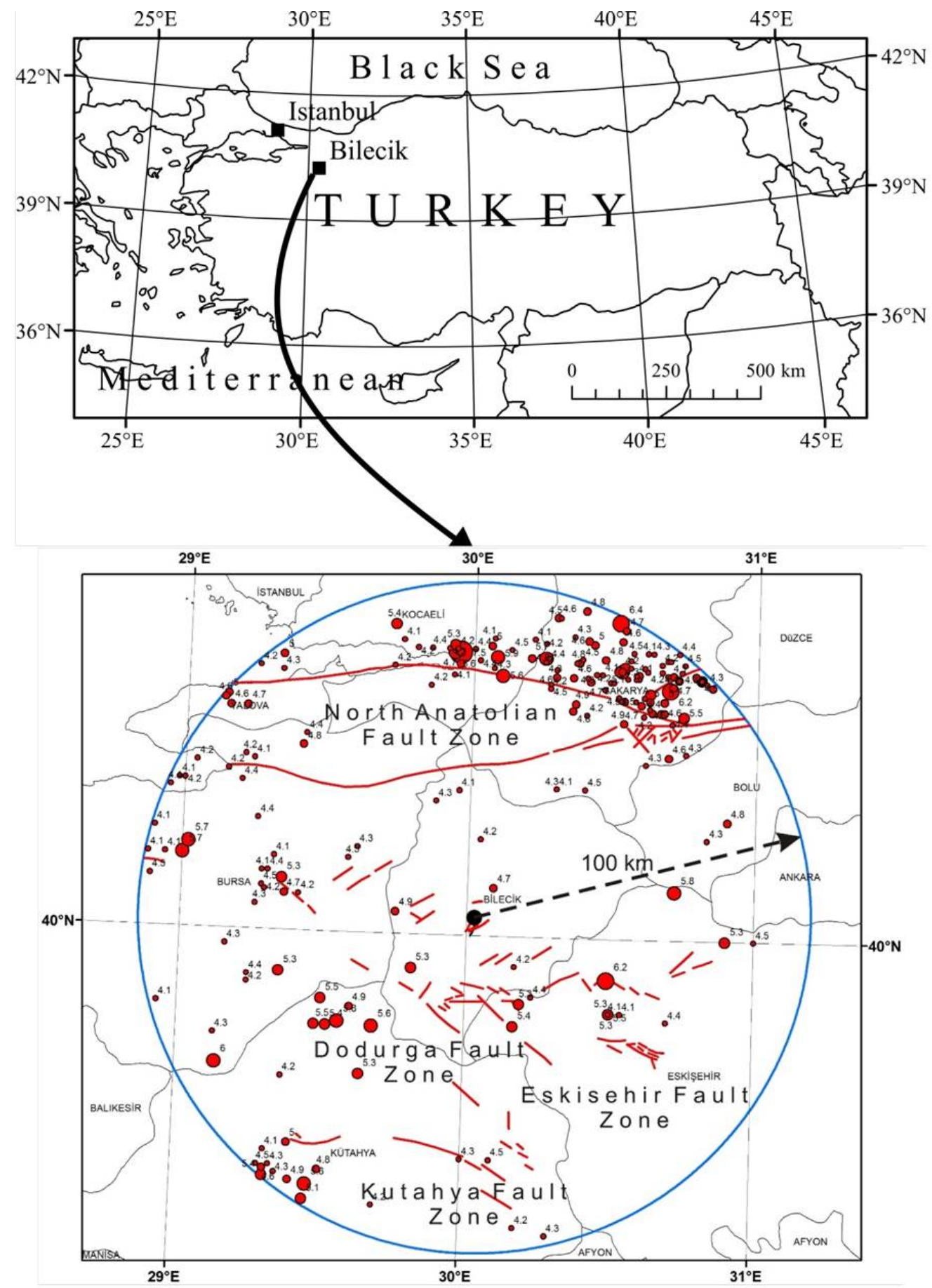

Figure 1. Geographic distributions of the earthquake data used in the study. 


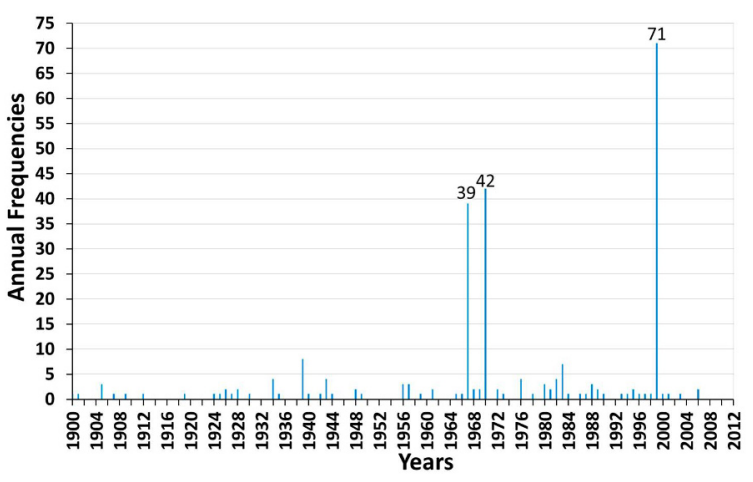

Figure 2. Histogram of annual frequencies of $M \geq 4.0$ earthquakes.

Poisson distributions and this result supports us to use HMM instead of using a Poisson process.

\section{Poisson Hidden Markov Model}

Markov models deal with the problems of the systems which are directly observable. In the real-world applications, the underlying system can be unobservable; but, can only be observed through another stochastic process. The evaluation of these partially observed systems is the major problem restricting the application areas of Markov models. HMM has a powerful and flexible mathematical structure to make statistical inferences on partially observed stochastic processes. It has been successfully applied to many diverse areas, particularly speech recognition [14-16], finance/econometrics [6, 1719], software reliability [20, 21], traffic engineering [22], Biology [23], language modeling [24, 25], metrology [2629], bioinformatics [30-33], biophysics/biochemistry [3436]. However, HMM has not as widely implemented as it should be in earthquake modeling. There have been few applications of HMM on earthquake problems [3739] Orfanogiannaki et al. [3, 4, 40-42]). Granat and Donnellan [37] used HMM as an unsupervised learning method for clustering of earthquakes and determining the classes of similar earthquakes in southern California region. Chambers et al. [38] applied HMM to model interevent times between earthquakes in New England. Ebel et al. [39] implemented HMM to extrapolate the future behavior of the past earthquake activity in California by using interevent earthquake times and the locations in which they occur. Orfanogiannaki et al. $[3,40,41]$ employed HMM to explain the frequencies of earthquakes occurred in specific time intervals for predicting the earthquake hazard. Wu [42] proposed a simple approach for HMM to decluster earthquakes in central and western regions of Japan. Chambers et al. [4] extended HMM to forecast the location of the earthquakes as well as interevent earthquake times in southern California and western Nevada. Let $T=\{0,1,2, \ldots, T\}$ a discrete-time horizon. HMM is a discrete-time bivariate stochastic process $(\mathrm{X}, \mathrm{Y})=\left(X_{t}, Y_{t}\right)_{t \in \mathrm{T}}[1,5,43]$

- $\mathrm{X}=\left(X_{t}\right)_{t \in \mathrm{T}}$ is a finite-state homogeneous Markov chain, which cannot be observable and thus referred as hidden process. $X_{t}$ is the state of the underlying system at time $t$.

- $\mathrm{Y}=\left(Y_{t}\right)_{t \in \mathrm{T}}$ is an observable process which depends on probabilistically Markov chain $X . \quad Y_{t}$ is the observation at time $t$. Observations can be discrete or continuous. In our study, a discrete sequence of observations is considered.

The probabilistic relationship between $\mathrm{X}$ and $\mathrm{Y}$ is given in Figure $3[5,44]$.

It is seen that at each point of time $t \in \mathrm{T}$, the conditional distribution of $Y_{t}$ given $X_{t}$ has a probability distribution coming from a specific family. $X$ is considered as the parameter process having the Markov property. According to unobservable parameter process, observations are generated by the state-dependent parametric distribution. In our study, HMM is used to forecast the frequencies of earthquakes for the next 35-year period. Therefore, the state-dependent parametric distribution is chosen as Poisson distribution. PHMM is a discrete-time bivariate stochastic process which consists of an unobserved finite state Markov chain $\mathrm{X}$ having $N$ states and an observed sequence of a non-negative integer valued stochastic process $Y$. On the condition that the states of the system $\mathrm{X}^{\top}=\left(X_{1}, \ldots, X_{T}\right)$ are known, $\mathrm{Y}^{\top}=\left(Y_{1}, \ldots, Y_{T}\right)$ are independent of each other. PHMM is characterized by the following five elements $[14,45,46]$

1. The Number of Hidden States $(N)$ : Each observation is derived from one of $N$ Poisson distributions. The rate parameters of Poisson distributions are called hidden states of finitestate homogeneous Markov chain X. For N-State PHMM, the finite set of $N$ states is defined as $=\lambda_{1}, \ldots, \lambda_{N}$.

2. The State Transition Probability Distribution: Which state will derive the next observation depends on which state derived the current observation through the state transition probability matrix $\mathrm{A}=\left[a_{i j}\right]_{N \times N}$. State transition probability to move from the state $\lambda_{i}$ at time $t$ to the state $\lambda_{j}$ at 


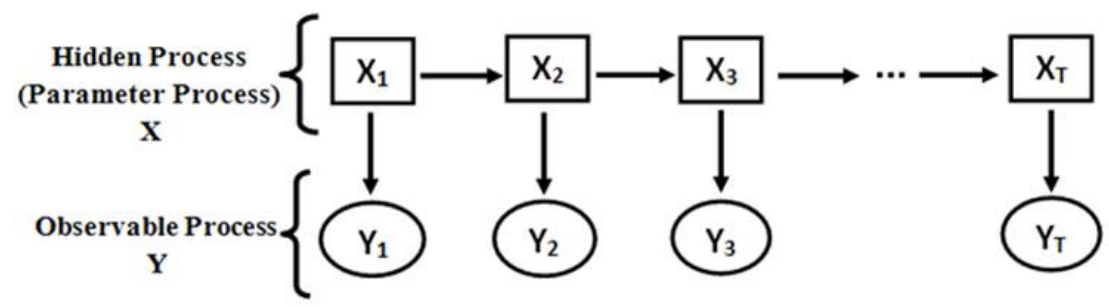

Figure 3. The dependence structure of a HMM [7].

time $t+1$ is given as $a_{i j}=P\left(X_{t+1}=\lambda_{j} \mid X_{t}=\lambda_{i}\right)$ for $\forall \lambda_{i}, \lambda_{j} \in \lambda$ and $t \in \mathrm{T}$.

3. The Domain of Observations per State: Each observation is non-negative integer valued.

4. The State-Dependent Probability Distribution: When the system is in state $\lambda_{i}$ at time $t$, the observation at that time is derived from Poisson distribution whose rate parameter is $\lambda_{i}$. The conditional distribution of $Y_{t}$ given $X_{t}$ is as follows

$$
\begin{array}{rlll}
P\left(Y_{t}=y \mid X_{t}=\lambda_{i}\right) & =e^{-\lambda_{i} \frac{\left(\lambda_{i}\right)^{y}}{y !}}, & & \text { if } y=0,1,2 \ldots \\
& =0, & & \text { otherwise }
\end{array}
$$

5. The Initial State Distribution: The starting state of the system is specified by the initial state probability vector $\pi=\left[\pi_{i}\right]_{1 \times N}$. Markov chain $\mathrm{X}$ starts at the state $\lambda_{i}$ with the probability $\pi_{i}=$ $P\left(X_{1}=\lambda_{i}\right)$ for $\forall \lambda_{i} \in \lambda$.

Given the above elements of PHMM, there are three fundamental problems of interest to be solved for realworld applications $[14,45,46]$

\section{Problem 1.}

The Evaluation Problem: Given the observation sequence $\mathrm{Y}^{\top}=\left(Y_{1} \ldots Y_{T}\right)$ and model parameters, what is the probability of the observation sequence? The solution can be seen as the likelihood $(L)$ of the model and thus provides the opportunity to choose the model which best explains the observations.

\section{Problem 2.}

The Decoding Problem: Given the observation sequence $Y^{\top}=\left(Y_{1} \ldots Y_{T}\right)$ and model parameters, what is the most likely state sequence $X^{\top}=\left(X_{1} \ldots X_{T}\right)$ which produces the given observation sequence? The hidden part of the model is uncovered by this problem.
Problem 3.

The Learning Problem: What are the optimal model parameters which maximize the probability of the given the observation sequence $\mathrm{Y}^{\top}=\left(Y_{1} \ldots Y_{T}\right)$ ? Due to the fact that the system is not directly observable, there is no analytic solution for this problem. Iterative methods are used for optimization. Here, EM algorithm is employed in order to find maximum likelihood estimates (MLEs) of PHMM parameters. EM algorithm does not guaranteed to converge at the global maximum and initials affect the MLEs of the parameters.

\section{Application of Poisson Hidden Markov Model and Results}

The problems given in section 3 are solved in this section. The frequencies of $M \geq 4$ earthquakes to occur in studied area for the next 35-year period are forecasted. The forecast results of PHMM are compared to those of homogeneous PP.

\subsection{Model Selection}

The first step is here to determine the number of hidden states for the selection procedure. Prior to the assessment of the earthquake hazard, the MLEs of PHMM parameters are estimated by EM algorithm. EM algorithm assumes that the number of hidden states are known and fixed. Therefore, first of all, the number of hidden states, which are really needed for the best explanation of the observations, must be determined. Clearly, the number of hidden states $(N)$ affects the quality of the statistical inferences. When likelihood of the model is taken into account, increasing $N$ always improves the fit of HMM. However, the improvement of the likelihood results in quadratic increase in the number of parameters $(p)$ and this improvement has to be balanced with the number of 


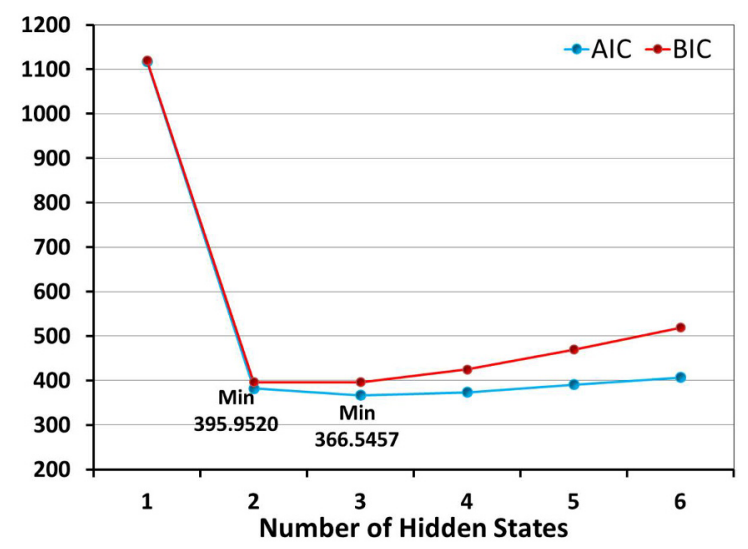

Figure 4. $\mathrm{AIC}$ and $\mathrm{BIC}$ for PHMMs with a different number of hidden states.

hidden states being fitted [1].

In order to compare HMMs with a fixed number of hidden states, Akaike's Information Criterion (AIC) and Bayesian Information Criterion (BIC) can be used as statistics for model selection. They are defined as follows $[47,48]$

$$
\begin{aligned}
& A I C=-2 \ln L+2 p \\
& B I C=-2 \ln L+p \ln (T)
\end{aligned}
$$

where $\ln L$ is the $\log$-likelihood of the fitted HMM, $p$ is the number of freely estimated parameters and $T$ is the number of observations. The parameters of PHMM with different number of hidden states are estimated by EM algorithm. 1000 iterations are realized for each of PHMMs in the study. Initial values which satisfy the log likelihoods as maximum are chosen from many sets of random starting values. In Figure 4, the calculated values of $A I C$ and BIC are given for PHMMs with the number of hidden states numbered from one to six. Clearly, one-state indicates a homogeneous PP.

According to AIC, 3-State PHMM is the best model. Contrary to AIC, 2-State PHMM is proposed by BIC. It is seen that there is no agreement between the two information criterias. In order to decide the most convenient number of the hidden states among the alternatives, the likelihood ratio test is applied in the study. The null and the alternative hypotheses are stated as follows:

$$
\begin{aligned}
& H_{0}: \text { The number of hidden states is } N \\
& H_{1}: \text { The number of hidden states is }(N+1)
\end{aligned}
$$

The likelihood ratio test statistic (LRT) is defined as below:

$$
\chi_{T \text { est }}=-2\left(\ln L_{0}-\ln L_{1}\right) \tilde{X}_{d f}=d f_{1}-d f_{0}
$$

where the $d f$ is the number of freely estimated parameters for the underlying PHMM [3]. The results for the possible number of hidden states are reported in Table 1.

According to P-values in Table 1, 3-State PHMM is decided as the best model at $1 \%$ level of significance. In 3-State PHMM, it is assumed that, an observation is generated from one of three different Poisson distribution at each point of time $t \in \mathrm{T}$. Its working principle of 3State PHMM is defined schematically in Figure 5.

In each counting time interval, earthquake occurrences depend on probabilistically Markov chain X. For instance, at time $t$, the observation $Y_{t}$ is derived from Poisson distribution whose rate parameter is $\lambda_{1}$.

The mean and variance of the frequencies of annual earthquake occurrences for 113 years (1900-2012) are calculated for six different models in the study and these statistics are given in Table 2. It indicates that the marginal distribution of 3-State PHMM meets overdispersed observations as well.

The observed numbers of years for each of the earthquake frequencies are compared to the expected numbers of frequencies obtained from 3-State PHMM and homogeneous PP. It can be seen that the observed and expected numbers of years are found to be similar for 3-State PHMM. A comparison of these two models for different annual earthquake frequencies is presented in Table 3. According to the table, there was no $M \geq 4$ earthquake occurrence observed for 56 years within 113 years and 3-State PHMM estimates that number as 56.5577. The observed and estimated numbers are very close. It indicates that there is an agreement of 3-State PHMM for the data and the model is suitable for further statistical analysis. As a result of EM algorithm, MLEs of 3-State PHMM parameters are given in Table 4.

\subsection{Prediction of Earthquake Hazard}

The pattern of the hidden states in the model for the next 35 years can be traced by calculating the state probability distribution. The probabilities of the hidden states for the coming 35 years are given in Figure 6. Here, the system in 2013 is assumed to have three states with the probabilities $0.86,0.12$ and 0.02 respectively. It can be seen in Figure 6 that the first hidden state remains as the most commonly seen state for the next 35 years. Therefore, it can be concluded that $M \geq 4$ earthquakes in the region will occur according to a Poisson distribution with the rate parameter, 0.4956 for the next 35 years. 
Table 1. Model Selection.

\begin{tabular}{llllll}
\hline Model & $\mathbf{p}$ & $-\operatorname{lnL}$ & $\mathbf{d f}$ & LRT & P-Value \\
\hline PP & 1 & 557.4164 & 3 & 742.5178 & $<0.01$ \\
2-State PHMM & 4 & 186.1575 & 5 & 27.7694 & $<0.01$ \\
3-State PHMM & 9 & 172.2728 & 7 & 9.2294 & $0.2366^{*}$ \\
4-State PHMM & 16 & 167.6581 & 9 & 2.667 & 0.9760 \\
5-State PHMM & 25 & 166.3246 & 11 & 7.8124 & 0.7300 \\
6-State PHMM & 36 & 162.4184 & & & \\
\hline
\end{tabular}

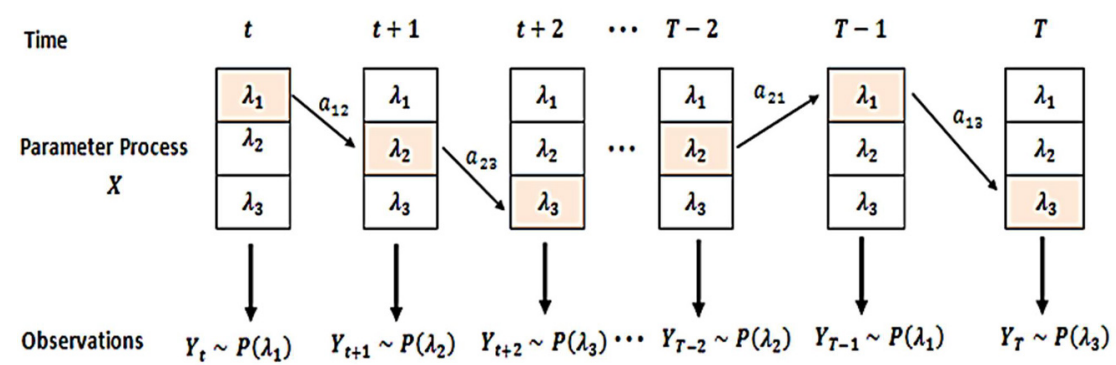

Figure 5. The working principle of 3-State PHMM.

Table 2. Comparison of Mean and Variances.

\begin{tabular}{|c|c|c|c|c|}
\hline \multirow{2}{*}{\multicolumn{2}{|c|}{ Observations }} & Mean & Variance & Variance-to-Mean Ratio \\
\hline & & 2.2301 & 71.9823 & 32.2776 \\
\hline \multirow{6}{*}{ 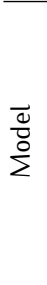 } & PS & 2.2301 & 2.2301 & 1.0000 \\
\hline & 2-State & 2.2419 & 66.7821 & 29.7882 \\
\hline & 3- State & 2.2460 & 67.6174 & 30.1057 \\
\hline & 4- State & 2.1210 & 62.0436 & 29.2521 \\
\hline & 5- State & 2.1577 & 63.8925 & 29.6114 \\
\hline & 6- State & 2.1577 & 69.0683 & 32.0101 \\
\hline
\end{tabular}

The distribution of frequencies for $M \geq 4$ earthquakes in the Zone is also forecasted for the next 35 years. According to data, the observed annual frequencies are ranged from zero to seventy-four earthquakes. The forecast distribution of the frequencies shows that the number of " 0 to 5 " earthquakes for the next 35 years are expected annually with the probability of 0.96 to 0.97. It strongly indicates that only a few numbers of earthquakes are expected to occur in the Zone between 2013 and 2047 and thus, the probability of having more than 5 earthquakes is considerably low. This result shows that earthquake hazard in the Zone is small, which is a natural result of the system is expected to be in first

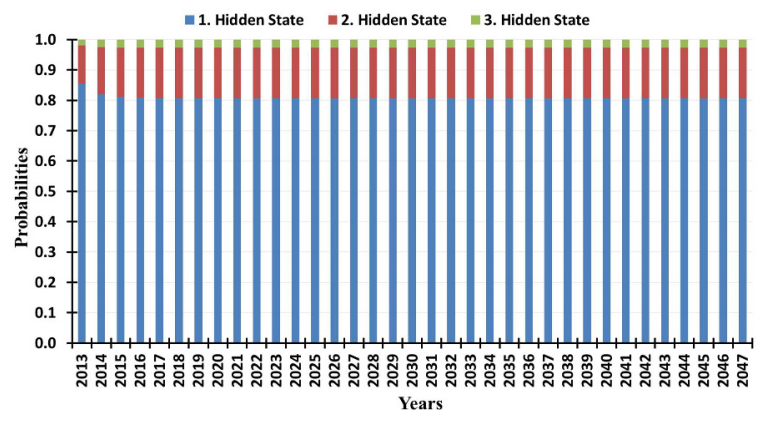

Figure 6. Hidden state prediction by 3-State PHMM for the Next 35 Years.

state $\left(\lambda_{1}=0.4956\right)$ for the next 35 -year period. The probabilities of earthquake frequencies ranging from 0 to 5 are shown in Figure 7. Due to the fact that Markov chain $\mathrm{X}$ reaches its stationary distribution $p i_{s}$ at 2022 , the next 10 years are reported only in Figure 7.

As a result of the fact that Markov chain $X$ reaches its stationary distribution within ten years, the expected annual frequencies of $M \geq 4$ earthquakes for the next 10 years and $95 \%$ confidence intervals (Cls) are calculated by 
Table 3. Model Agreement Comparison for 3-State PHMM and PP.

\begin{tabular}{llll}
\hline $\begin{array}{l}\text { Annual Earthquake } \\
\text { Frequency }\end{array}$ & $\begin{array}{l}\text { Observed Numbers } \\
\text { of Years }\end{array}$ & 3-State PSMM & PP \\
\hline 0 & 56 & 56.5577 & 12.1496 \\
1 & 32 & 30.4497 & 27.0948 \\
2 & 11 & 11.1066 & 30.2119 \\
3 & 5 & 5.3278 & 22.4584 \\
4 & 4 & 3.2296 & 12.521 \\
5 & 0 & 1.8323 & 5.5846 \\
6 & 0 & 0.8929 & 2.0757 \\
7 & 1 & 0.3749 & 0.6613 \\
8 & 1 & 0.1379 & 0.1843 \\
9 & 0 & 0.0451 & 0.0457 \\
10 & 0 & 0.0133 & 0.0102 \\
$\geq 11$ & 3 & 3.0323 & 0.0025 \\
TOTAL & 113 & 113 & 113 \\
\hline
\end{tabular}

Table 4. Parameter Estimations by EM Algorithm.

\begin{tabular}{ll}
\hline Parameters & MLEs \\
\hline Poisson Rate Parameters & $\lambda=\left[\begin{array}{lll}\lambda_{1} & \lambda_{2} & \lambda_{3}\end{array}\right]=\left[\begin{array}{lll}0.4956 & 2.9425 & 50.6667\end{array}\right]$ \\
State Transition Probability Distribution & $A=\left[\begin{array}{lll}0.8591 & 0.1219 & 0.0190 \\
0.5743 & 0.3568 & 0.0689 \\
0.6858 & 0.3142 & 0.000\end{array}\right]$ \\
Initial State Distribution & $\pi_{0}=\left[\begin{array}{lll}0 & 0 & 0\end{array}\right]$ \\
Stationary State Distribution & $\pi_{S}=\left[\begin{array}{lll}0.8072 & 0.1660 & 0.0268\end{array}\right]$ \\
\hline
\end{tabular}

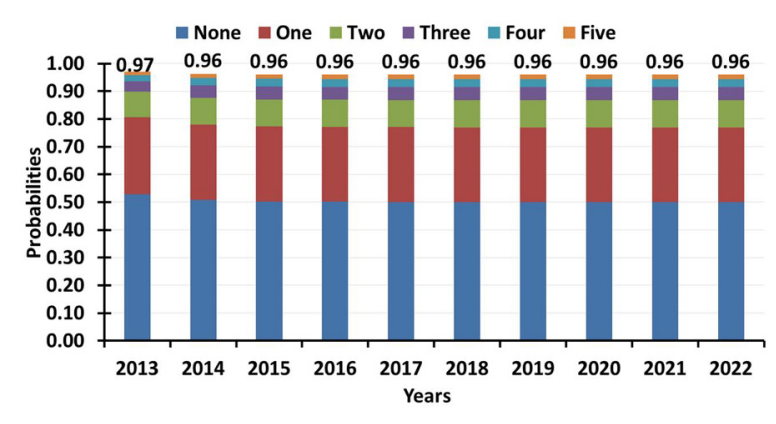

Figure 7. The probabilities of earthquake frequencies in the range 0 to 5 for $2013-2022$.

forecast frequency distribution. They are given in Table 5. It is seen that approximately two earthquakes per year are expected to occur in the Zone within the next 35 years.
The expected frequencies in Table 1 show that 3-State PHMM explains the earthquake data better than PP. In the following part of the study, 3-State PHMM and PP are compared in detailed by the predicting the earthquake hazards. The distributions of earthquake frequencies for the next seven years are examined for the both models. Although the probabilities are calculated for the frequencies ranging from zero to seventy-one, Figure 8 includes the considerably high frequencies and their related probabilities obtained from two models for each year.

Figure $8 \mathrm{~A}$ shows that 3 -State $\mathrm{PHMM}$ and $\mathrm{PP}$ predict that less than 6 earthquakes will occur in a year with the probability of $97 \%$. Even if the two models forecast the same probability, they differ in the amount of probabilities related to frequencies. Especially, the absence of an earthquake is more likely to occur in 3-State PHMM $(52.8 \%)$ than PP $(10.8 \%)$. It is seen in Figure $8 \mathrm{~B}$ that the probabilities of having less than 6 earthquakes are $91.4 \%$ for 3 -State PHMM and $71 \%$ for PP respectively. 


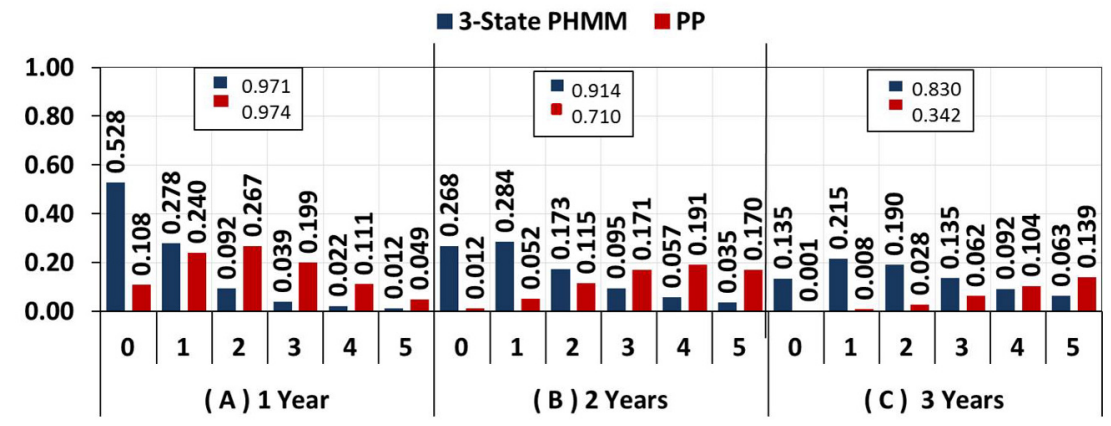

Figure 8. Earthquake hazard within " 1 to 3 " Years.

Table 5. Expected Annual Frequencies of $M \geq 4$ Earthquakes for the Next 10 Years.

\begin{tabular}{lll}
\hline Year & \multicolumn{2}{l}{$\begin{array}{l}\text { Expected } \\
\text { Annual Frequency }\end{array}$} \\
\hline 2013 & 1.785 & {$[0.181-3.389]$} \\
2014 & 2.121 & {$[0.326-3.916]$} \\
2015 & 2.211 & {$[0.369-4.054]$} \\
2016 & 2.235 & {$[0.381-4.090]$} \\
2017 & 2.242 & {$[0.384-4.100]$} \\
2018 & 2.244 & {$[0.385-4.103]$} \\
2019 & 2.244 & {$[0.385-4.103]$} \\
2020 & 2.244 & {$[0.385-4.104]$} \\
2021 & 2.244 & {$[0.385-4.104]$} \\
2022 & 2.244 & {$[0.385-4.104]$} \\
\hline
\end{tabular}

Our model has the highest probabilities for the lower frequencies, while PP has the lowest probabilities for the lower frequencies (for example $1.2 \%$ and $5.2 \%$ are the probabilities for "0" and "1" earthquakes within 2 years). For 3-State PHMM, "0 to 5" earthquakes are expected with the probability of $83 \%$ in three years time. However, less than 6 earthquakes in three years are less likely to occur for PP (34.2\%).

The earthquake hazards over the 10 years rapidly increase in PP. The traditional model tends to predict higher frequencies than it should be in fact. For example, the probability of less than eleven earthquakes decreases from $71.5 \%$ to $9.2 \%$ within the four years starting from 2015 (Figure 9). However, there is no sudden decrease observed in 3-State PHMM (Figure 9). Most likely, less than eleven earthquakes will occur within the years $(89.1 \%, 84.9 \%, 79.8 \%$ and $73.8 \%$ respectively).
Expected numbers of $M \geq 4$ earthquakes in the next 1 to 7 years and their related $95 \% \mathrm{Cls}$ are calculated for the two models and Table 6 shows the results. The expected numbers found from the models are not significantly different, but $95 \%$ Cls have quite different. Earthquake data indicates strong over-dispersion. As a natural result of over-dispersion, Cls are expected to be wider than they should be. In Table 6, the ranges of $\mathrm{Cls}$ for PP are found narrower than those for 3-State PHMM as they are expected.

\section{Conclusion}

The use of HMM to predict earthquake hazard is carried out by Granat and Donnellan [37], Chambers et al. [38], Ebel et al. [39], Orfanogiannaki et al. [3, 40, 41], Wu [42] and Chambers et al. [4]. All previous studies about earthquake data modeling by HMM except Orfanogiannaki et al. $[3,40,41]$ are based on the continuous earthquake data and interevent earthquake times. The forecasting methodology followed in the present study is similar to Orfanogiannaki et al. [3]. PHMM is applied to model the annual frequencies of earthquakes occurred within a radius of $100 \mathrm{~km}$ area centered on Bilecik, in the period between January 1900 and December 2012, with magnitudes $(M)$ at least 4.0 by using data provided from the catalogs of Boğaziçi University, Kandilli Observatory and Earthquake Research Institute [9]. This study is the first application of PHMM to the Turkish earthquake data except a short version of this study presented by Can et al. [7]. In addition to Orfanogiannaki et al. [3], the forecast results of PHMM are compared to those of homogeneous PP. Predictions of hidden states for the next 35 years show that earthquakes occurred in compliance with $P\left(\lambda_{1}=\right.$ 


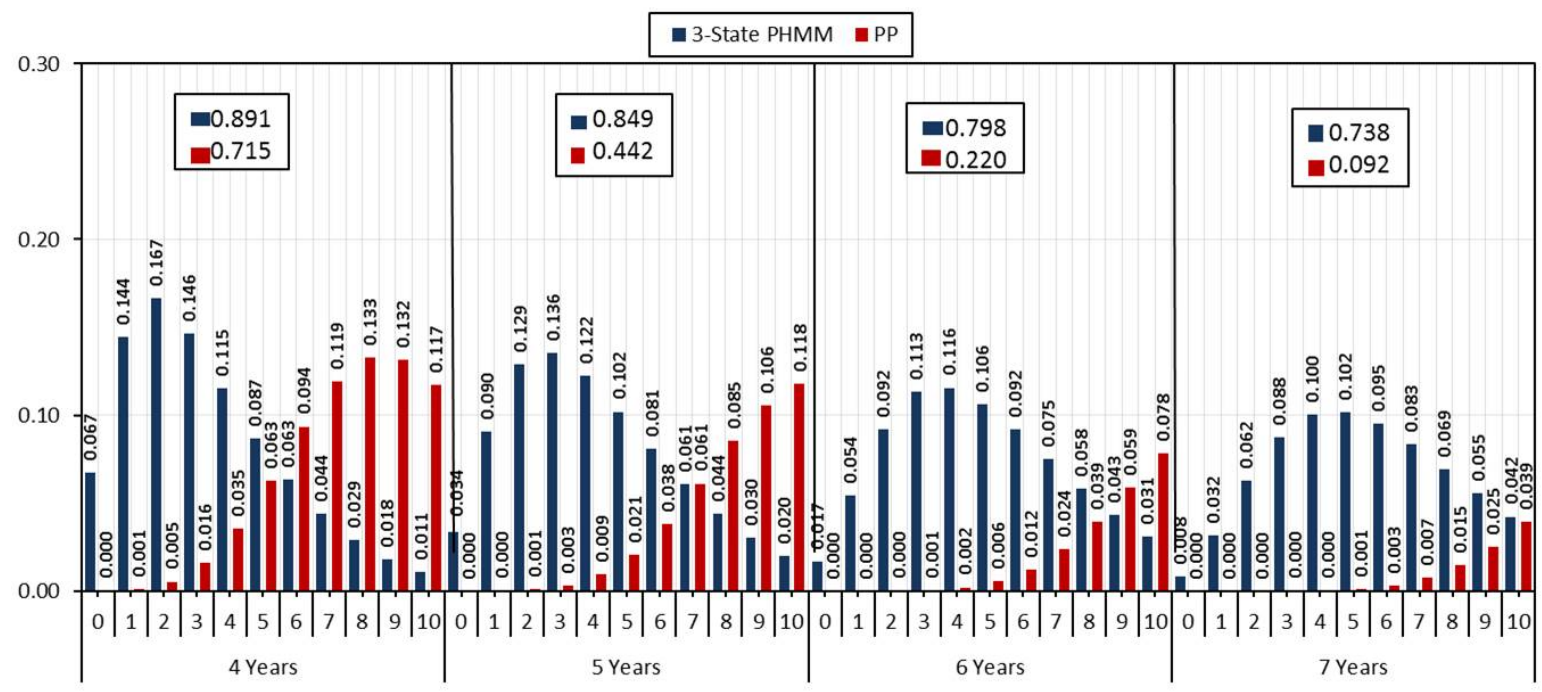

Figure 9. Earthquake hazard within " 4 to 7 " Years.

Table 6. Expected Numbers of Earthquakes over the years.

\begin{tabular}{lrrrr}
\hline \multirow{2}{*}{\begin{tabular}{l} 
Time Horizon \\
\cline { 2 - 5 } (within ... years)
\end{tabular}} & $\begin{array}{r}\text { 3-State PHMM } \\
\text { Expected }\end{array}$ & \%95 Cl & $\begin{array}{r}\text { Expected } \\
\text { Frequency }\end{array}$ & \%95 Cl \\
\hline 1 & Frequency & {$[0.149-3.416]$} & 2.230 & {$[1.885-2.575]$} \\
2 & 1.782 & {$[1.448-6.243]$} & 4.460 & {$[3.972-4.948]$} \\
3 & 3.846 & {$[2.979-8.864]$} & 6.690 & {$[6.093-7.288]$} \\
4 & 5.921 & {$[4.577-11.294]$} & 8.920 & {$[8.230-9.610]$} \\
5 & 7.936 & {$[6.179-13.553]$} & 11.150 & {$[10.379-11.922]$} \\
6 & 9.866 & {$[7.750-15.654]$} & 13.381 & {$[12.536-14.225]$} \\
7 & 11.702 & {$[9.266-17.603]$} & 15.611 & {$[14.698-16.523]$} \\
\hline
\end{tabular}

0.50) are more likely to happen than those occurred in compliance with $P\left(\lambda_{2}=2.94\right)$ and $P\left(\lambda_{3}=50.67\right)$. Also, 0 to 5 numbers of $M \geq 4.0$ earthquakes are expected to occur with the probability not less than 0.96 in the studied area for the next 35 years. As for the modeling and forecasting performance of HMM and PP, it is concluded that PHMM produces meaningful results to predict the annual frequencies for earthquakes of a particular magnitude.

One of the most important parameters used in the design of large engineering constructions is the horizontal acceleration resulted from earthquakes. The magnitude of an earthquake is one of the main parameters used to calculate the maximum horizontal acceleration. Therefore, the use of the results obtained by PHMM provides crucial information for the prediction of the momentum which may come upon the engineering structures. For the future studies, taking into consideration of HMM in earthquake hazard problems makes serious contributions to design more economical and safer engineering structures.

\section{References}

[1] Zucchini W. and MacDonald I. L., Hidden Markov Models for Time Series: An Introduction Using $\mathrm{R}$, Chapman \& Hall/CRC Monographs on Statistics \& Applied Probability, Boca Raton, USA, 2009 
[2] Baum L. E. and Petrie T., Statistical Inference for Probabilistic Functions of Finite State Markov Chains, The Annals of Mathematical Statistics, 37(1), 1966, 1554-1563

[3] Orfanogiannaki K., Karlis D. and Papadopoulos G. A., Identifying Seismicity Levels via Poisson Hidden Markov Models, Pure and Applied Geophysics, 167, 2010, 919-931

[4] Chambers D. W., Baglivo J. A., Ebel J. E. and Kafka A. L., Earthquake Forecasting Using Hidden Markov Models, Pure and Applied Geophysics, 169, 2012, 625-639

[5] Cappe O., Moulines E. and Ryden T., Inference in Hidden Markov Models, Springer Science+Business Media, New York, USA, 2005

[6] MacDonald I. L. and Zucchini W., Hidden Markov Models and Other Models for Discrete-Valued Time Series, Chapman \& Hall/CRC Monographs on Statistics \& Applied Probability, London, UK, 1997

[7] Can C. E., Ergun, G., Gokceoglu C., Bilecik çevresinde deprem tehlikesinin saklı Markov modeli ile tahmini. Proceedings of the Second Turkey Earthquake Engineering and Seismology Conference, 25-27 Eylül 2013 - MKÜ - Hatay, Turkey (in Turkish), 2013

[8] Ocakoglu F., Acikalin S., Gokceoglu C., Nefeslioglu H. A., Sonmez, H., Back-analysis of the source of the 1956 Eskisehir Earthquake using attenuation equation and damage data, Bull Eng Geol Environ, 66, 2007, 353-360

[9] UIDM, Boğaziçi University, Kandilli Observatory and Earthquake Research Institute, Web Address: http: //www.koeri.boun.edu.tr/, 2013

[10] Boore D. M., Joyner W. B., Fumal T. E., Equations for estimating horizontal response spectra and peak acceleration from western North American earthquakes: a summary of recent work, Seismol. Res. Lett., 68 (1), 1997, 128-153

[11] Campbell K. W., Empirical near source attenuation relationships for horizontal and vertical components of peak ground acceleration, peak ground velocity, and pseudo-absolute acceleration response spectra, Seismol. Res. Lett., 68(1), 1997, 154-179

[12] Sadigh K., Chang C. Y., Egan J. A., Makdisi F., Youngs R. R., Attenuation relationships for shallow crustal earthquakes based on California strong motion data, Seismol. Res. Lett., 1(68), 1997, 180-189

[13] Ulusay R., Tuncay E., Sonmez H., Gokceoglu C., An attenuation relationship based on Turkish strong motion data and iso-acceleration map of Turkey, Engineering Geology, 74, 2004, 265-291

[14] Rabiner L. R., A Tutorial on Hidden Markov Models and Selected Applications in Speech Recognition, Proceedings of the IEEE, 77(2), 1989, 257-285

[15] Rabiner L. R. and Juang B. -H.,Fundamentals of Speech Recognition, Prentice-Hall, New Jersey, USA, 1993

[16] Jelinek F., Statistical Methods for Speech Recognition, MIT Pres, USA, 1997

[17] Hamilton J. D., Time Series Analysis. Princeton University Press, New Jersey, USA, 1994

[18] Rydén T., Teräsvirta and Asbrink S., Stylized facts of daily returns series and the Hidden Markov Model, Journal of Applied Econometrics, 13, 1998, 217-244

[19] Kim C. -J. and Nelson, C. R., State-Space Models with Regime Switching: Classical and GibbsSampling Approaches with Applications, MIT Press, USA, 1999

[20] A., Ruggeri F. and Soyer R., A Bayesian Hidden Markov Model for Imperfect Debugging, Reability Engineering and System Safety, 103, 2012, 11-21

[21] Landon J., Özekici S. and Soyer R., A Markov Modulated Poisson Model for Software Reability, European Journal of Operational Research, 229, 2013, 404-410

[22] Dainotti A., Pescapé A., Rossi P. S., Palmieri F., and Ventre G., Internet Traffic Modelling by Means of Hidden Markov Models, Computer Networks, 52(14), 2008, 2645-2662

[23] Leroux B. G. and Puterman M. L., MaximumPenalized-Likelihood Estimation for Independent and Markov-Dependent Mixture Models, Biometrics, 48(2), 1992, 545-558

[24] Cave R. L. and Neuwirth L. P., Hidden Markov Models for English, In Proceedings of the Symposium on the Application of Hidden Markov Models to Text and Speech (Ferguson J. D. Editor), Princeton, New Jersey: IDA-CRD, 16-56, 1980

[25] Charniak E., Statistical Language Learning, MIT Press, Massachusetts, 170, 1993

[26] Zucchini W. and Guttorp P., A Hidden Markov Model for Space-Time Precipitation, Water Resources Research, 27(8), 1991, 1917-1923

[27] Sansom J., A Hidden Markov Model for Rainfall Using Breakpoint Data, Journal of Climate, 11, 1998, 42-53

[28] Bellone E., Hughes J. P. and Guttorp P., A hidden Markov model for downscaling synoptic atmospheric patterns to precipitation amounts, Climate Research, 15, 2000, 1-12

[29] Robertson A. W., Kirshner S. and Smyth P., Downscaling of Daily Rainfall Occurrence over Northeast Brazil Using a Hidden Markov Model, Journal of Climate, 17, 2004, 4407-4424

[30] Baldi P., Hunkapiller T., Chauvin Y. and McClure, 
M. A., Hidden Markov Models of Biological Primary Sequence Infromation, In Proceedings of National Academy of Science USA, Vol. 91, 1994, 1059-1063

[31] Krogh A., Brown M., Mian I. S., Sjölander K. and Haussler D., Hidden Markov models in computational biology: Applications to protein modeling, Journal of Molecular Biology, 235, 1994, 1501-1531

[32] Durbin R., Eddy S., Krogh A. and Mitchison G., Biological Sequence Analysis: Probabilistic Models of Proteins and Nucleic Acids. Cambridge University Press, Cambridge, England, 1998

[33] Koski T., Hidden Markov Models for Bioinformatics. Kluwer Academic Publishers, USA, 2001

[34] Fredkin D. R. and Rice J. A., Bayesian Restoration of Single-channel Patch Clamp Recordings, Biometrics, 48(2), 1992, 427-448

[35] Fredkin D. R. and Rice J. A., Maximum Likelihood Estimation and Identification Directly from Singlechannel Recordings, Proceedings: Biological Science, 249, 1992, 125-132

[36] Ball F. G., Cai Y., Kadane J. B. and O'Hagan A., Bayesian Inference for Ion-Channel Gating Mechanisms Directly from Single-Channel Recordings, Using Markov Chain Monte Carlo. Proceedings Royal Society London A, 455, 1999, 2879-2932

[37] Granat R. and Donnellan A., A Hidden Morkov Model Based Tool for Geophysical Data Exploration, Pure and Applied Geophysics, 159 (10), 2002, 2271-2283

[38] Chambers D. W., Ebel J. E., Kafka A. L. and Baglivo J. A., Hidden Markov Approach to Modeling Interevent Earthquake Times, Eos. Trans. AGU, 84(46), 2003, Fall Meet. Suppl., Abstract S52F-0179

[39] Ebel J. E., Chambers D. W., Kafka A. L. and Baglivo J. A., Non-Poissonian Earthquake Clustering and the Hidden Markov Model as Bases for Earthquake Forecasting in California, Seismological Research Letters, 78 (1), 2007, 57-65

[40] Orfanogiannaki K., Karlis D. and Papadopoulos G. A.,Identification of Temporal Patterns in the Seismicity of Sumatra Ruprue Zone Using Poisson Hidden Markov Models, Geophysical Research Abstracts, Volume 8, 2006

[41] Orfanogiannaki K., Karlis D. and Papadopoulos G. A., Application of Poisson Hidden Markov Models on Foreshock Sequence: The case of Samos, October 2005 and Zakynthos, April 2006, Earthquakes, XIIth Int. Conf. Appl. Stochastic Models and Data Analysis, Chania, Crete, Greece, 2007

[42] Wu Z., A Hidden Markov Model for Earthquake Declustering, Journal of Geophysical Research, 115, 2010

[43] Ephraim Y. and Merhav N., Hidden Markov Processes, IEEE Transactions On Information Theory, 48:6, 2002, 1518-1569

[44] Ghahramani Z., An Introduction to Hidden Markov Models and Bayesian Networks, International Journal of Pattern Recognition and artificial Intelligence, 15:1, 2001, 9-42

[45] Alpaydin E., Introduction to Machine Machine Learning, The MIT Press, Cambridge, USA, 2010

[46] Ibe O. C., Markov Process for Stochasting Modelling, Elsevier Aacademic Press, California, USA, 2010

[47] Akaike H., A New Look at the Statistical Model Identification, IEEE Transactions on Automatic Control, AC-19, 1974, 716-723

[48] Schwarz G., Estimating the Dimension of a Model, Annals of Statistics, 6, 1978, 461-464 